\title{
Is a ureteral stent required before flexible ureteroscopy?
}

\author{
Qiyi Hu ${ }^{1 \#}$, Yongjian Ji ${ }^{2 \#}$, Zhu Wang ${ }^{1}$, Yulin Lai ${ }^{1}$, Qiong Deng ${ }^{1}$, Jianwen Zhang ${ }^{1}$, Hongliang Wang ${ }^{1}$, \\ Hui Liang ${ }^{1}$, Hongjun Zhao $^{2}$
}

${ }^{1}$ Department of Urology, People's Hospital of Longhua, Southern Medical University, Shenzhen, China; ${ }^{2}$ Department of Urology, Weifang People's Hospital, Weifang, China

Contributions: (I) Conception and design: All authors; (II) Administrative support: None; (III) Provision of study materials or patients: None; (IV) Collection and assembly of data: None; (V) Data analysis and interpretation: W Zhu, Y Lai, Q Deng, J Zhang, H Wang, H Liang; (VI) Manuscript writing: All authors; (VII) Final approval of manuscript: All authors.

\#These authors contributed equally to this work.

Correspondence to: Hongjun Zhao. Department of Urology, Weifang People's Hospital, No. 151, Guangwen Street, Kuiwen District, Weifang 261041, China. Email: doctorzhao2000@126.com; Hui Liang. Department of Urology, People’s Hospital of Longhua Shenzhen, Southern Medical University, No. 38, Jianshe Road, Longhua, Shenzhen 518109, China. Email: dr.lianghui@aliyun.com.

Background: To retrospectively evaluate the therapeutic effectiveness and safety of flexible ureteroscopes without preoperative ureteral stent placement.

Methods: A total of 243 patients who had undergone flexible ureteroscopy (fURS) lithotripsy were reviewed. The patients were divided into two groups: 119 patients without preoperative ureteral stent placement were set as group A; and the remaining 124 patients who received preoperative ureteral stent placement were set as group B. The operative time, length of hospital stay, hospital costs, stone-free rates (SFRs), complications, and re-operation rates of the two groups were respectively compared.

Results: Pairwise analysis indicated the following: the average operative time of group A was longer than that of group B $(66.53 \pm 10.19$ versus $59.85 \pm 9.85 \mathrm{~min}, \mathrm{P}=0.0001)$, the average length of hospital stay of group A was considerably shorter than that of group B $(6.56 \pm 0.90$ versus $10.67 \pm 1.50 \mathrm{~d}, \mathrm{P}=0.0001)$, the SFRs of group A were significantly lower than those of group B at 3 days postoperatively $(36.1 \%, 43 / 119$ versus $51.6 \%, 64 / 124, \mathrm{P}=0.0034)$, and the average hospital costs were substantially lower in group A than those in group $\mathrm{B}(18,756$ versus $23,450 \mathrm{RMB}, \mathrm{P}=0.0001)$. However, there were no notable differences observed in the following: SFRs between the groups at 1 month postoperatively $(84.0 \%, 100 / 119$ of group A versus $85.5 \%$, $106 / 124$ of group B, $\mathrm{P}=0.895)$, complications rates $(20.1 \%, 26 / 124$ in group A versus 20.1\%, 23/114 in group $\mathrm{B}, \mathrm{P}=0.597)$, and re-operation rates $(15.1 \%, 18 / 119$ in group A versus $16.9 \%, 21 / 124$ in group $\mathrm{B}, \mathrm{P}=0.558)$.

Conclusions: These results indicated that fURS without preoperative ureteral stent placement is safe and effective for the treatment of upper urinary calculi.

Keywords: Flexible ureteroscopy (fURS); upper urinary calculi; ureteral stent; urology

Submitted Sep 24, 2020. Accepted for publication Dec 13, 2020.

doi: $10.21037 /$ tau-20-1458

View this article at: http://dx.doi.org/10.21037/tau-20-1458

\section{Introduction}

Urolithiasis is the third most common dysfunction of the urinary system, and affects $10-15 \%$ of the general population globally $(1,2)$. The incidence of urolithiasis has increased over the past decades. Most patients who suffer from kidney stones are between 30 and 50 years old, and the recurrence rate is about $35-50 \%$ (3). Most stones that are $4 \mathrm{~mm}$ in diameter or smaller are spontaneously and asymptomatically eliminated from the body via urination. However, stones measuring $5 \mathrm{~mm}$ or larger are not typically removed from the body through the urine, and could cause severe pain, urethral obstruction, hydronephrosis, infection, and hemorrhage, and thus must be referred to a urologist 
$(4,5)$. The diagnosis and treatment of urolithiasis is still a major challenge for urologists $(4,6)$.

Over the recent decades, a variety of strategies have been developed and utilized for the surgical management of kidney stones, benefitting from many technological advancements (7). Generally, urologic surgical approaches are increasingly becoming less invasive and mainly include extracorporeal shockwave lithotripsy (ESWL), ureteroscopy (URS), and percutaneous nephrolithotomy (PCNL). The choice of surgical modality for ureteral and renal calculi are optimized individually on the basis of stone size, location, and composition (if known), as well as local expertise and patient preference (8). However, current European Association of Urology (EAU) guidelines recommend URS and PCNL endourological procedures, and ESWL has lost its place as the primary modality for active ureteral stone treatment, despite still being effective (1). At present, due to developing technologies in the endoscopic armamentarium, flexible ureteroscopy (fURS) has gained in popularity and become the primary therapeutic option for the treatment of renal stones $(<2 \mathrm{~cm})$ because of its high stone-free rates (SFRs) and low morbidity (8-11).

Some investigations have demonstrated that ureteral stent (Double-J tube) placement has been widely applied before fURS to dilate obstructed ureters and allow for better access to the upper tract during ureteroscopy, which could improve the efficiency of the procedure and significantly enhance the SFRs $(12,13)$. Conversely, several studies have reported that there were no significant differences in the SFRs between pre-stented and non-stented patients who undergo fURS (12-15). Preoperative ureteral stents may also cause additional compilations, including urinary tract perforation, urosepsis, ureter avulsion, and bleeding with transfusion $(12,13,16-18)$. Thus, it remains controversial whether a ureteral stent is required before fURS for patients without obvious ureteral strictures $(19,20)$.

In this study, we performed a retrospective review of patients who had undergone fURS with and without preoperative stent placement. We compared and evaluated the differences in operative time, SFRs, re-operation rates, length of hospital stay, and hospital costs between the patient groups, and aimed to investigate whether a ureteral stent is required before undergoing fURS for patients suffering from upper urinary calculi.

We present the following article in accordance with the STROBE reporting checklist (available at http://dx.doi. org/10.21037/tau-20-1458).

\section{Methods}

\section{Patient demographics}

All procedures performed in this study involving human participants were in accordance with the Declaration of Helsinki (as revised in 2013). This research was approved by the Research Ethics Boards of the People's Hospital of Longhua Shenzhen. Written informed consent was obtained from the patients beforehand.

The inclusion criteria were as follows: (I) patients with stone diameters less than $2 \mathrm{~cm}$ located in unilateral upper ureteral, unilateral renal pelvis, or both unilateral ureteral and renal pelvis; and (II) patients who had undergone uncomplicated fURS with or without preoperative ureteral stent placement. The exclusion criteria were as follows: (I) patients with isolated kidney or renal insufficiency; (II) patients with ureteral malformations or ureteral stenosis; (III) patients with preoperative urinary tract infection; (IV) patients with severe cardiopulmonary dysfunction; (V) patients with bilateral urinary tract stones; (VI) patients who could not be placed in the lithotomy position; and (VII) patients with severe urethral strictures.

The included patients were classified into two groups depending on whether they had a ureteral stent placed prior to fURS. All patients had received ultrasonography, plain film of kidney-ureter-bladder (KUB), and computed tomography urography (CTU) examination before the operation. All routine experimental examinations, including urine analysis, urine culture, renal function testing, and coagulation profiling were performed prior to fURS. Clinical parameters such as gender, age, and comorbidities were retrieved and summarized in Table 1.

\section{Surgical procedure}

All surgical procedures were performed by four experienced urologists (QH, HL, JZ, and HW) using a standardized technique with last-generation flexible ureteroscopes. The decision to stent (F5 Double-J tube, Bard Medical, Covington, USA) or not to stent the patient prior to fURS was determined according to the discretion of the urologist. All patients had received general anesthesia and were placed in the lithotomy position. Subsequent ureteroscopic procedures were performed with zebra guidewires, including a safety wire (Sensor, Boston Scientific, Natick, USA). A ureteral access sheath was routinely used, and was typically $35 \mathrm{~cm}$ for women and $45 \mathrm{~cm}$ for men, with the 
Table 1 Patient demographics and stone characteristics

\begin{tabular}{|c|c|c|c|c|c|c|c|c|c|c|}
\hline Group & \multicolumn{2}{|c|}{ Gender } & \multicolumn{2}{|c|}{ Laterality of stones } & Age (year) & $\begin{array}{l}\text { Size of stones } \\
(\mathrm{mm})\end{array}$ & \multicolumn{2}{|c|}{ Hydronephrosis } & \multicolumn{2}{|c|}{ Location of stone } \\
\hline Group A (non-stented) & 74 & 45 & 72 & 47 & $46.39 \pm 9.55$ & $14.52 \pm 3.32$ & 61 & 58 & 62 & 57 \\
\hline Group B (pre-stented) & 78 & 46 & 68 & 56 & $42.99 \pm 11.23$ & $15.54 \pm 3.38$ & 75 & 49 & 57 & 67 \\
\hline$P$ value & \multicolumn{2}{|c|}{0.426} & \multicolumn{2}{|c|}{0.193} & 0.940 & 0.898 & \multicolumn{2}{|r|}{0.066} & \multicolumn{2}{|c|}{0.078} \\
\hline
\end{tabular}

sheath diameter determined according to the discretion of the surgeon.

The conventional F5 Double-J tube and F16 catheters were routinely placed postoperatively for all patients. Ultrasonic and KUB examinations were performed preoperatively, and at 1-month and 3-months after fURS to determine the residual calculus (representative images are shown in Figure 1). All patients were discharged from hospital in an outpatient setting.

\section{Analyzed parameters}

We retrospectively reviewed data from patient documentation, KUB, CTU, and operation reports. Patient demographics and stone characteristics were analyzed. We compared parameters including operative time, re-operation rates, SFRs, length of hospital stay, hospital costs, and complications rates between both groups.

\section{Statistical analysis}

The SPSS20 statistical software was utilized for statistical analysis. Groups were compared using the Mann-Whitney $\mathrm{U}$ test. Categorical variables were compared using Fisher's exact test. $\mathrm{P}<0.05$ was considered to be statistically significant.

\section{Results}

Among the 245 patients who underwent fURS, 119 patients did not receive preoperative ureteral stents (group A), while the remaining 124 patients had received preoperative ureteral stent placement (group B). The two groups were matched for age $(\mathrm{P}=0.940)$, gender $(\mathrm{P}=0.426)$, laterality of stones $(\mathrm{P}=0.193)$, location of stones $(\mathrm{P}=0.078)$, and size of stones $(\mathrm{P}=0.898)$. The patient demographic and stone characteristics of the two groups are summarized in Table 1.

A successful stone-free status was achieved if all endoscopically or radiographically visible stone fragments had been completely removed. The SFRs were determined and compared at 3-days and 1-month following fURS. The group B patients who had received preoperative stent placement showed a significantly higher SFR at 3-days after fURS than that of non-stented patients $(51.6 \%, \mathrm{n}=64 / 124$ vs. $36.1 \%, \mathrm{n}=43 / 119, \mathrm{P}=0.0034)$. However, there were no notable differences in the SFRs between the two groups at 1-month after fURS (84.0\% vs. 85.5\%, $\mathrm{P}=0.895)$ (Figure 2).

The total operative time was reduced by approximately 6 min in group B patients with preoperative ureteral stent placement (59.85 vs. $66.53 \mathrm{~min}, \mathrm{P}=0.001$, Figure $3 A$ ). Meanwhile, the length of hospital stay was longer in the pre-stented group than that of non-stented group (10.67 vs. 6.56 days, $\mathrm{P}=0.001$, Figure $3 B$ ). No major complications were encountered during stent placement or ureteroscopy in presented patients in this study. We observed no significant differences in the primary complications, including chills, fever, urinary tract infection, and the need for analgesia, between the pre-stented and non-stented groups $(21.3 \%$ vs. $20.1 \%, P=0.597)$. Furthermore, there were no significant differences in the re-operation rates between the groups $(\mathrm{P}=0.558)$. The hospital costs of the pre-stented patient group were markedly higher than that of non-stented group $(23,450$ vs. 18,756 RMB, $\mathrm{P}=0.0001$, Figure 4). Details of the postoperative parameters comparison between the prestented and non-stented groups are shown in Table 2.

\section{Discussion}

Benefitting from technical advancements and the introduction of a wide range of disposables, fURS has gained in popularity in recent years. The most recent EAU guidelines recommend fURS as the primary therapeutic option in the treatment of upper urinary tract stones $<2 \mathrm{~cm}$ $(9,21,22)$. Yet, the requirement for ureteral stent placement prior to fURS remains a topic of debate.

In the present study, we found that preoperative stenting could significantly increase the SFRs at 3-days after fURS, which seems to be consistent with several previous reports 

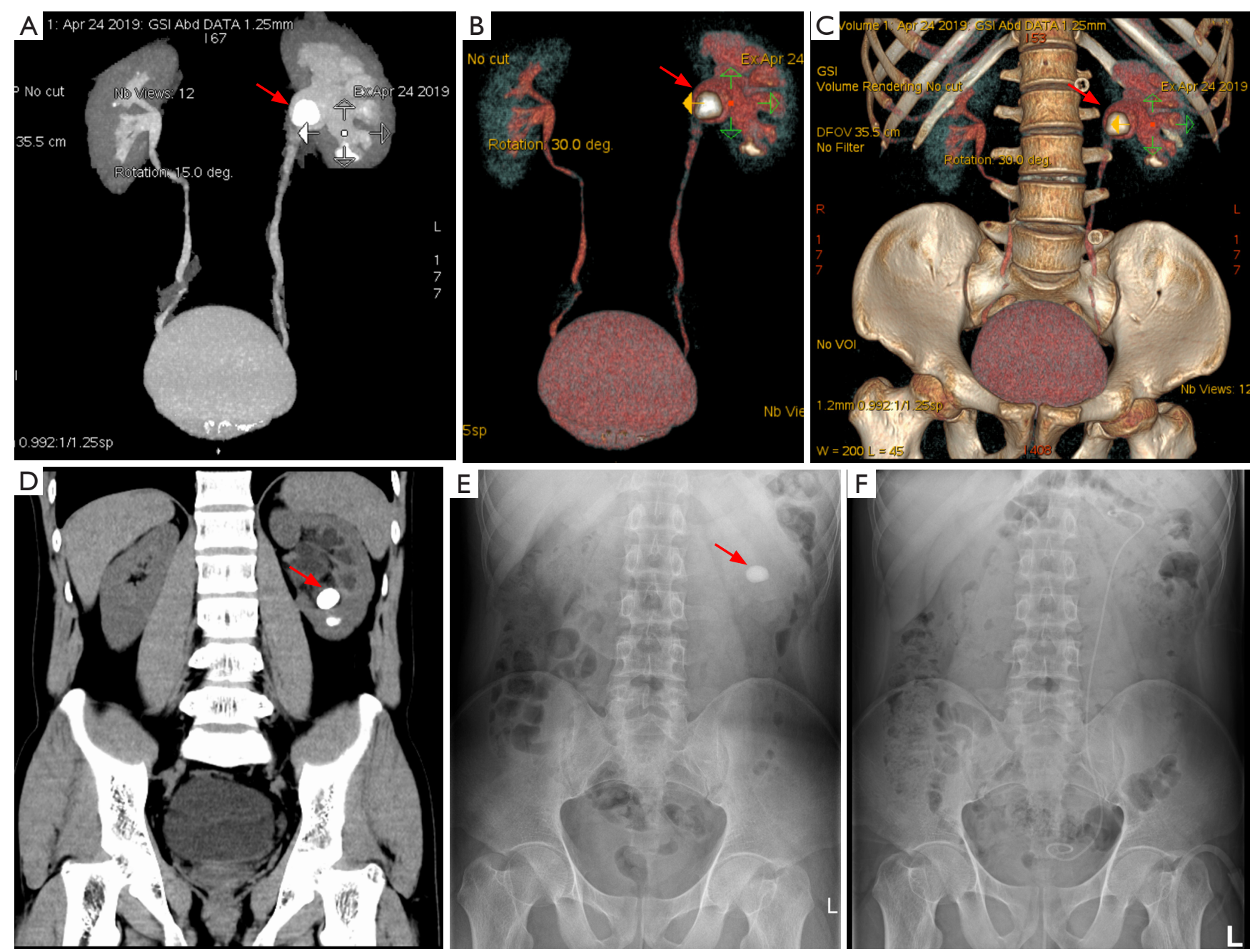

Figure 1 Representative images of CTU and KUB examination before and after fURS. (A-C) Representative images of 3D reconstruction before fURS; (D) representative image of magnetic resonance imaging (MRI) examination before fURS; (E) representative image of KUB examination at 1-week after fURS; (F) representative image of KUB examination at 1-month after fURS. The red arrows indicate lesion locations. CTU, computed tomography urography; KUB, kidney-ureter-bladder; fURS, flexible ureteroscopy.

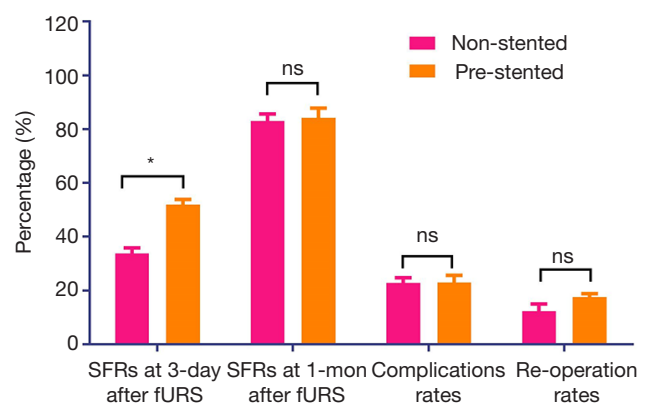

Figure 2 Comparison of SFRs, complications rates, and reoperation rates between the non-stented and pre-stented groups. *, $\mathrm{P}<0.05$; ns, no significance. SFR, stone-free rate; fURS, flexible ureteroscopy.
$(12,17)$. However, the SFRs of stented and non-stented patients were quite similar with no significant differences at 3 -months after surgery. In addition, the operative time was significantly reduced (by approximately $6 \mathrm{~min}$ ) compared to that in the non-stented group. This could be attributed to the dilation of the ureter by preoperative stent placement, which enhanced the success rate of ureteroscopic access and provided improved vision of the ureteral tract (23). However, the reduced operative time makes little sense in clinical practice. Interestingly, several studies have claimed that the SFRs were significant higher in pre-stented patients who underwent fURS with no major complications $(13,19,24-26)$. This difference might be due to an overall 

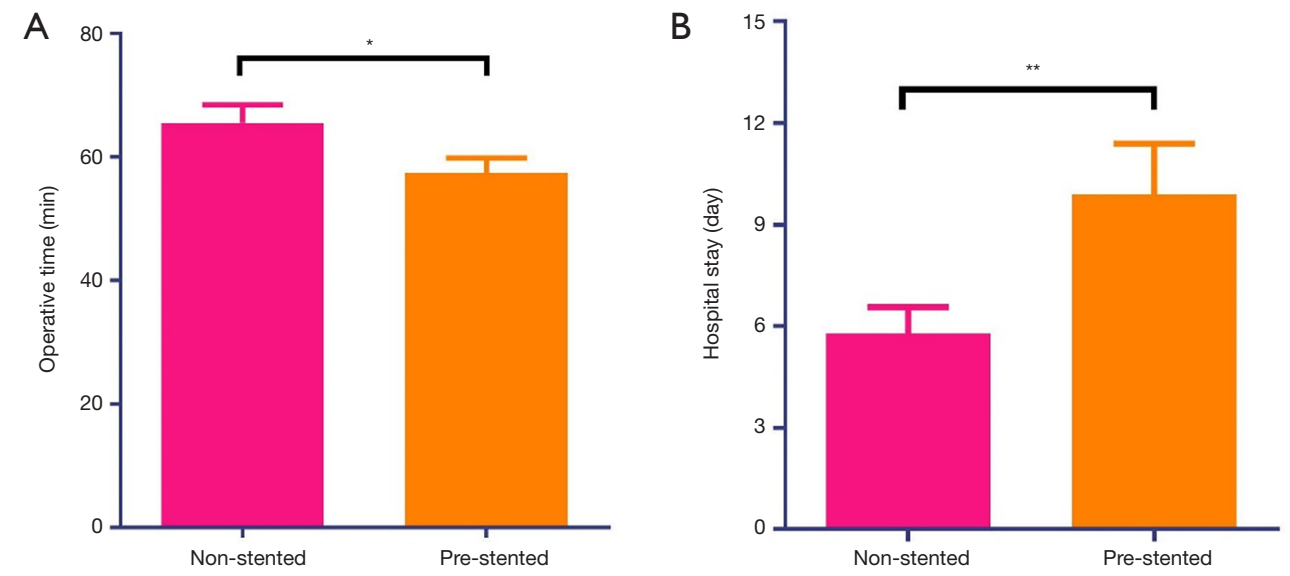

Figure 3 Comparison of operative time and length of hospital stay between the non-stented and pre-stented groups. *, $\mathrm{P}<0.05$; **, $\mathrm{P}<0.001$.

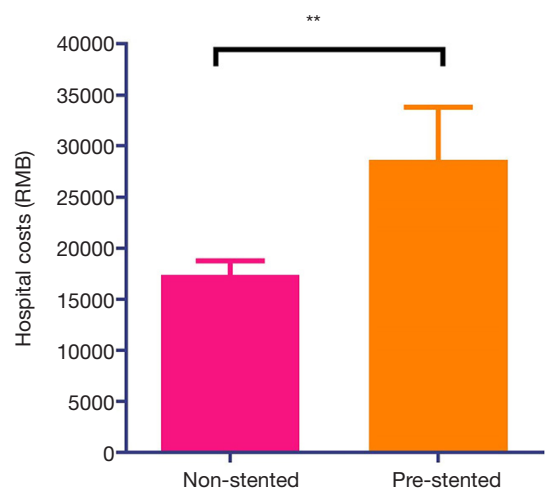

Figure 4 Comparison of hospital costs between the non-stented and pre-stented groups. ${ }^{* *}, \mathrm{P}<0.001$.

lower stone size and upper stone location in our series.

According to the results of our retrospective analysis, the complication and re-operation rates were not significantly different between preoperative stented and non-stented groups, which was similar to previous findings $(12,14,17,27)$. No major complications were encountered during stent placement or ureteroscopy in presented patients in this study. Other complications, such as postoperative bleeding and ureteral mucosal injury, which might be caused during intraoperative flexible ureteroscope sheath insertion and Holmium laser lithotripsy $(28,29)$, were not included in this study.

Although preoperative stent placement is significant in the treatment of upper ureteral calculi, it results in an increased hospital stay and hospital costs. This is likely attributable to the extra preoperative stenting procedure, which will inevitably extend the hospital stay and increase hospital costs. For patients with ureteral stone street, where large residual stones cannot be excreted via ESWL, rigid ureteroscopy or secondary fURS should be performed subsequently (30). However, this is not related to the preoperative placement of Double-J tubes.

There are some limitations in our study that should be noted. Firstly, the major limitation of our study was that the consensus for Double-J stent placement was not identified preoperatively. The urologists decided whether or not to stent independently, without guidelines or previous agreement. The preoperative stent size and duration of SFRs was unclear. Secondly, the number of included patients included is not large enough, owing to the fact that this study was performed in one single hospital. Thirdly, considering that this is a retrospective study, potential inherent selection bias cannot entirely be excluded.

In summary, we found that preoperative ureteral stent placement can help to decrease the operative time and improve the SFRs for patients undergoing fURS, but not significantly so. Preoperative stenting extended the length of hospital stay and increased hospital costs, but had no influence on complication and re-operation rates. These findings lead to the conclusion that preoperative ureteral stent placement before fURS for the treatment for upper urinary calculi is not reasonable. However, given the limitations of this study, more high-quality investigations including prospective randomized clinical trials are required to confirm our findings. 
Table 2 Comparison of postoperative parameters between the two groups

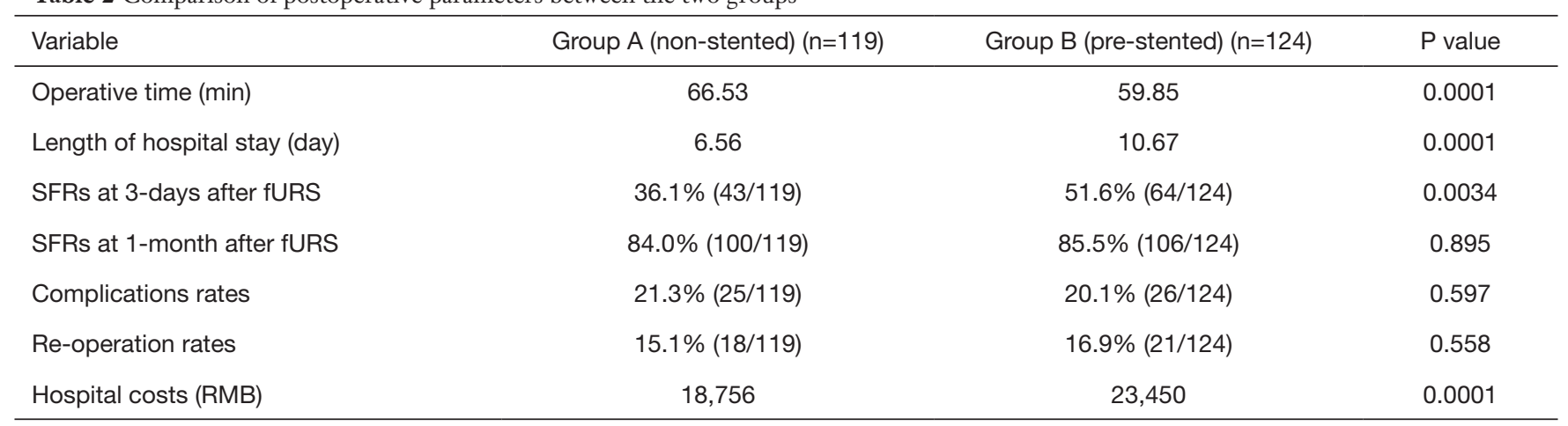

fURS, flexible ureteroscopy. SFRs, stone-free rates.

\section{Acknowledgments}

Funding: This study was financially supported by the Shenzhen Science and Technology Program (Basic Research Project, No. JCYJ20180228163919346 to ZW) and the Shenzhen Longhua Science and Technology Innovation Fund (2017009 to ZW, 20150925A0410013 to HL).

\section{Footnote}

Reporting Checklist: The authors have completed the STROBE reporting checklist. Available at http://dx.doi. org/10.21037/tau-20-1458

Data Sharing Statement: Available at http://dx.doi. org/10.21037/tau-20-1458

Conflicts of Interest: All authors have completed the ICMJE uniform disclosure form (available at http://dx.doi. org/10.21037/tau-20-1458). The authors have no conflicts of interest to declare.

Ethical Statement: The authors are accountable for all aspects of the work in ensuring that questions related to the accuracy or integrity of any part of the work are appropriately investigated and resolved. All procedures performed in this study involving human participants were in accordance with the Declaration of Helsinki (as revised in 2013). This research was approved by the Research Ethics Boards of the People's Hospital of Longhua Shenzhen. Written informed consent was obtained from the patients beforehand.

Open Access Statement: This is an Open Access article distributed in accordance with the Creative Commons Attribution-NonCommercial-NoDerivs 4.0 International License (CC BY-NC-ND 4.0), which permits the noncommercial replication and distribution of the article with the strict proviso that no changes or edits are made and the original work is properly cited (including links to both the formal publication through the relevant DOI and the license). See: https://creativecommons.org/licenses/by-nc-nd/4.0/.

\section{References}

1. Turk C, Petrik A, Sarica K, et al. EAU Guidelines on Interventional Treatment for Urolithiasis. Eur Urol 2016;69:475-82.

2. Sorokin I, Mamoulakis C, Miyazawa K, et al. Epidemiology of stone disease across the world. World J Urol 2017;35:1301-20.

3. Pearle MS, Calhoun EA, Curhan GC, et al. Urologic diseases in America project: urolithiasis. J Urol 2005;173:848-57.

4. Hosking DH, Erickson SB, Van den Berg CJ, et al. The stone clinic effect in patients with idiopathic calcium urolithiasis. J Urol 1983;130:1115-8.

5. Assadi F, Moghtaderi M. Preventive Kidney Stones: Continue Medical Education. Int J Prev Med 2017;8:67.

6. Lopez M, Hoppe B. History, epidemiology and regional diversities of urolithiasis. Pediatr Nephrol 2010;25:49-59.

7. Skolarikos A. Medical treatment of urinary stones. Curr Opin Urol 2018;28:403-7.

8. Giusti G, Proietti S, Villa L, et al. Current Standard Technique for Modern Flexible Ureteroscopy: Tips and Tricks. Eur Urol 2016;70:188-94.

9. Doizi S, Traxer O. Flexible ureteroscopy: technique, tips and tricks. Urolithiasis 2018;46:47-58. 
10. Raheem OA, Khandwala YS, Sur RL, et al. Burden of Urolithiasis: Trends in Prevalence, Treatments, and Costs. Eur Urol Focus 2017;3:18-26.

11. Yasui T, Iguchi M, Suzuki S, et al. Prevalence and epidemiological characteristics of urolithiasis in Japan: national trends between 1965 and 2005. Urology 2008;71:209-13.

12. Lumma PP, Schneider P, Strauss A, et al. Impact of ureteral stenting prior to ureterorenoscopy on stone-free rates and complications. World J Urol 2013;31:855-9.

13. Netsch C, Knipper S, Bach T, et al. Impact of preoperative ureteral stenting on stone-free rates of ureteroscopy for nephroureterolithiasis: a matched-paired analysis of 286 patients. Urology 2012;80:1214-9.

14. Assimos D, Crisci A, Culkin D, et al. Preoperative JJ stent placement in ureteric and renal stone treatment: results from the Clinical Research Office of Endourological Society (CROES) ureteroscopy (URS) Global Study. BJU Int 2016;117:648-54.

15. Chu L, Sternberg KM, Averch TD. Preoperative stenting decreases operative time and reoperative rates of ureteroscopy. J Endourol 2011;25:751-4.

16. Zhang J, Xu C, He D, et al. Flexible ureteroscopy for renal stone without preoperative ureteral stenting shows good prognosis. PeerJ 2016;4:e2728.

17. Yang Y, Tang Y, Bai Y, et al. Preoperative double-J stent placement can improve the stone-free rate for patients undergoing ureteroscopic lithotripsy: a systematic review and meta-analysis. Urolithiasis 2018;46:493-9.

18. Miernik A, Wilhelm K, Ardelt PU, et al. Standardized flexible ureteroscopic technique to improve stone-free rates. Urology 2012;80:1198-202.

19. Astroza G, Catalan M, Consigliere L, et al. Is a ureteral stent required after use of ureteral access sheath in

Cite this article as: Hu Q, Ji Y, Wang Z, Lai Y, Deng Q, Zhang J, Wang $\mathrm{H}$, Liang $\mathrm{H}$, Zhao $\mathrm{H}$. Is a ureteral stent required before flexible ureteroscopy? Transl Androl Urol 2020;9(6):2723-2729. doi: 10.21037/tau-20-1458 presented patients who undergo flexible ureteroscopy? Cent European J Urol 2017;70:88-92.

20. Torricelli FC, De S, Hinck B, et al. Flexible ureteroscopy with a ureteral access sheath: when to stent? Urology 2014;83:278-81.

21. Keller EX, Doizi S, Villa L, et al. Which flexible ureteroscope is the best for upper tract urothelial carcinoma treatment? World J Urol 2019;37:2325-33.

22. Inoue T, Okada S, Hamamoto $S$, et al. Current trends and pitfalls in endoscopic treatment of urolithiasis. Int J Urol 2018;25:121-33.

23. Jones BJ, Ryan PC, Lyons O, et al. Use of the double pigtail stent in stone retrieval following unsuccessful ureteroscopy. Br J Urol 1990;66:254-6.

24. Al-Qahtani SM, Letendre J, Thomas A, et al. Which ureteral access sheath is compatible with your flexible ureteroscope? J Endourol 2014;28:286-90.

25. Cho SY. Current status of flexible ureteroscopy in urology. Korean J Urol 2015;56:680-8.

26. Rubenstein RA, Zhao LC, Loeb S, et al. Prestenting improves ureteroscopic stone-free rates. J Endourol 2007;21:1277-80.

27. Kawahara T, Ito $\mathrm{H}$, Terao $\mathrm{H}$, et al. Preoperative stenting for ureteroscopic lithotripsy for a large renal stone. Int J Urol 2012;19:881-5.

28. Black KM, Aldoukhi AH, Ghani KR. A Users Guide to Holmium Laser Lithotripsy Settings in the Modern Era. Front Surg 2019;6:48.

29. Wang XK, Jiang ZQ, Tan J, et al. Thermal effect of holmium laser lithotripsy under ureteroscopy. Chin Med J (Engl) 2019;132:2004-7.

30. Sullere A, Sureka B, Khera PS. 'Stone street' ureter. Abdom Radiol (NY) 2018;43:2204-5.

(English Language Editor: A. Kassem) 